\title{
„Nutritional Risk Screening 2002“ in der Hand des Pneumologen
}

\author{
“Nutritional Risk Screening 2002"- in Clinical Pneumology
}

Autoren

Institute
C. Priegnitz ${ }^{1}$, W. Galetke ${ }^{2}$, M. Treml' ${ }^{1}$, W. J. Randerath ${ }^{1}$

${ }^{1}$ Institut für Pneumologie an der Universität Witten Herdecke (Prof. Dr. med. Winfried J. Randerath), Krankenhaus Bethanien gGmbH, Solingen

${ }^{2}$ Krankenhaus der Augustinerinnen, Köln eingereicht 18.2 .2014 akzeptiert nach Revision 23.4.2014

Bibliografie

Dol http://dx.doi.org/ 10.1055/s-0034-1365737 Online-Publikation: 5.6.2014 Pneumologie 2014; 68: 478-482 (c) Georg Thieme Verlag KG Stuttgart · New York ISSN 0934-8387

Korrespondenzadresse Dr. med. Christina Priegnitz Institut für Pneumologie an der Universität Witten Herdecke Krankenhaus Bethanien gGmbH Aufderhöher Str. 169-175 42699 Solingen christina.priegnitz@klinikbethanien.de

\section{Zusammenfassung}

$\nabla$

Mangelernährung (ME) ist ein Problem bei Krankenhauspatienten und stellt einen Risikofaktor für Morbidität und Mortalität dar.

Ziel der Studie war es, die Häufigkeit von Unterernährung und das Risiko einer ME bei konsekutiven, stationären Patienten einer pneumologischen Fachklinik zu überprüfen.

Die Unterernährung wurde mittels Body-MassIndex (BMI), das ME-Risiko mittels „Nutritional Risk Screening 2002“" (NRS) überprüft und in Bezug auf Alter, Geschlecht, Dauer des Krankenhausaufenthaltes und der pneumologischen Erkrankung untersucht.

Das NRS wurde bei 680 von 705 Patienten überprüft. Es zeigte bei 14,3\% ein ME-Risiko. Bei 689 Patienten wurde der BMI bestimmt, bei 2,5\% lag eine Unterernährung vor. Bei den über 65-jährigen $(n=326)$ war das NRS in $19,6 \%$ der Fälle positiv, der BMI $\left(<18,5 \mathrm{~kg} / \mathrm{m}^{2}\right)$ in $1,5 \%$. Alter stellte einen signifikanten Risikofaktor für ein ME-Risiko dar (OR 1,054 pro Lebensjahr), das Geschlecht nicht.

In einer Subanalyse zeigte sich bei Pneumonieund COPD-Patienten ein ME-Risiko in 23\% bzw. $16 \%$. Tumorpatienten waren häufiger von einem ME-Risiko betroffen, Patienten mit einer schlafbezogenen Atmungsstörung (SBAS) seltener (OR 2,33 bei Tumor, OR 0,04 bei SBAS). Ein ME-Risiko war mit einem signifikant längeren Krankenhausaufenthalt assoziiert (10,2 $\pm 9,5$ vs. 5,4 $\pm 6,0$ Tage). Das NRS erweitert neben dem BMI die Beurteilung einer ME.

\section{Einleitung}

$\nabla$

Mangelernährung ist ein häufiges Gesundheitsproblem stationärer Patienten (20-50\%) [1-5] und entspricht einem relevanten Risikofaktor für Morbidität und Mortalität [6-8]. Mangelernährte Patienten verbleiben länger stationär und werden

\section{Abstract \\ $\nabla$}

Malnutrition is a frequent problem for hospitalized patients. It is a relevant risk factor for morbidity and mortality.

The aim of this study was to detect undernutrition and the risk of malnutrition (RM) in patients admitted to a university-affiliated respiratory care clinic. Undernutrition was assessed by body mass index (BMI $\left.<18.5 \mathrm{~kg} / \mathrm{m}^{2}\right)$ and RM by using the "Nutritional Risk Screening 2002" (NRS 2002) in 705 consecutive patients (BMI: measured in 689 patients/NRS: 680 patients assessed).

Data was analysed with regard to age, sex, length of hospital stay as well as underlying pneumological disorders. In $14.3 \%$ of 680 patients, RM was detected by NRS. In $2.5 \%$ out of 689 patients, undernutrition was identified by BMI. In patients older than 65 years $(n=365)$, these numbers were $19.6 \%$ (NRS) and $1.5 \%\left(\mathrm{BMI}<18.5 \mathrm{~kg} / \mathrm{m}^{2}\right)$. Age was a significant risk factor for RM (OR 1.054 per year). Gender, however, was not associated with undernutrition or RM.

In a sub-analysis, RM was more frequent in patients with pneumonia and chronic obstructive lung disease (23\% and $16 \%$, respectively).

Patients with cancer were more frequently at RM as compared to patients with sleep-disordered breathing (OR: 2.33 in cancer, OR: 0.04 in sleepdisordered breathing). RM was associated with a significant increase in length of hospital stay ( $10.2 \pm 9.5$ vs. $5.4 \pm 6.0$ days $)$.

Besides the BMI, the NRS provides a valid tool for screening patients at RM.

häufiger wiederaufgenommen $[9,10]$. In einer deutschen Multizenterstudie an stationären Patienten wurde eine Mangelernährung anhand des Body-Mass-Indexes (BMI) bei 4,1\% der untersuchten Patienten festgestellt [3]. Der Anteil an Frauen sowie älteren Patienten war dabei deutlich erhöht [3]. Standardmäßig wird der BMI zur 
Tab. 1 Geschlecht, Alter, BMI, Anteil NRS-positiver Patienten (NRS-Score $\geq 3$ ) und Anteil untergewichtiger Patienten (BMI $\left.<18,5 \mathrm{~kg} / \mathrm{m}^{2}\right)$ in den verschiedenen Erkrankungsgruppen. Für Alter und BMI sind jeweils Mittelwert \pm Standardabweichung sowie die Anzahl vorliegender Messwerte angegeben.

\begin{tabular}{|c|c|c|c|c|c|}
\hline $\begin{array}{l}\text { Pneumologische } \\
\text { Erkrankungsgruppe }\end{array}$ & Geschlecht & Alter (Jahre) & BMI $\left(\mathrm{kg} / \mathrm{m}^{2}\right)$ & $\begin{array}{l}\text { NRS-positive Patienten } \\
\text { (NRS Score } \geq 3 \text { ) }\end{array}$ & $\begin{array}{l}\text { Untergewichtige Patienten } \\
\left(\mathrm{BMI}<18,5 \mathrm{~kg} / \mathrm{m}^{2}\right)\end{array}$ \\
\hline $\begin{array}{l}\text { Schlafbezogene } \\
\text { Atmungsstörungen }\end{array}$ & $\begin{array}{l}71 w, 214 m \\
(n=285)\end{array}$ & $\begin{array}{l}58,6 \pm 12,8 \\
(n=285)\end{array}$ & $\begin{array}{l}33,4 \pm 7,7 \\
(n=285)\end{array}$ & $3 / 265(1 \%)$ & $1 / 285(0 \%)$ \\
\hline Tumorerkrankung & $\begin{array}{l}41 w, 62 m \\
(n=103)\end{array}$ & $\begin{array}{l}65,0 \pm 10,8 \\
(n=103)\end{array}$ & $\begin{array}{l}25,9 \pm 5,4 \\
(n=103)\end{array}$ & $38 / 101(38 \%)$ & $5 / 103(5 \%)$ \\
\hline $\begin{array}{l}\text { Obstruktive } \\
\text { Atemwegserkrankung }\end{array}$ & $\begin{array}{l}73 w, 101 m \\
(n=174)\end{array}$ & $\begin{array}{l}64,9 \pm 12,2 \\
(n=174)\end{array}$ & $\begin{array}{l}27,8 \pm 6,5 \\
(n=166)\end{array}$ & $28 / 168(17 \%)$ & $7 / 166(4 \%)$ \\
\hline Sonstige & $\begin{array}{l}72 w, 71 m \\
(n=143)\end{array}$ & $\begin{array}{l}61,4 \pm 16,1 \\
(n=143)\end{array}$ & $\begin{array}{l}28,3 \pm 7,1 \\
(n=135)\end{array}$ & $28 / 136(21 \%)$ & $4 / 135(3 \%)$ \\
\hline Gesamt & $\begin{array}{l}258 w, 447 m \\
(n=705)\end{array}$ & $\begin{array}{l}61,7 \pm 13,4 \\
(n=705)\end{array}$ & $\begin{array}{l}29,9 \pm 7,6 \\
(n=689)\end{array}$ & $97 / 680(14 \%)$ & $17 / 689(3 \%)$ \\
\hline
\end{tabular}

$\mathrm{w}=$ weiblich, $\mathrm{m}=$ männlich, $\mathrm{BMI}=$ Body-Mass-Index, NRS= Nutritional Risk Screening

Gewichtsbeurteilung genutzt, wobei Unterernährung gemäß WHO ab einem BMI-Wert unter $18,5 \mathrm{~kg} / \mathrm{m}^{2}$ definiert ist.

Das Nutritional Risk Screening 2002 (NRS) wurde als Screeninginstrument für die Risikoabschätzung einer Mangelernährung von Kondrup validiert [2].

Almeida verglich das NRS mit anderen Instrumenten zur Bewertung eines ME-Risikos bei chirurgischen Patienten. Sie konnte zeigen, dass das NRS valide, handhabbar und einfach in der Anwendung ist [11]. So lagen die Werte für Sensitivität und Spezifität zwischen 80 und $90 \%$. Ähnliches galt für den negativ und positiv prädiktiven Wert (80 und 90\%) [11]. Das NRS überzeugte insbesondere bei stationären Patienten in zahlreichen randomisierten klinischen Studien [12].

Patienten mit pneumologischen Grunderkrankungen weisen per se eine hohe Letalität auf:

Bei Pneumoniepatienten schwanken die Zahlen zwischen 10 und $50 \%$ [13-15]. Untergewicht $\left(\mathrm{BMI}<18,5 \mathrm{~kg} / \mathrm{m}^{2}\right)$ wurde als ein weiterer relevanter Risikofaktor für das Entstehen einer ambulant erworbenen Pneumonie bestätigt [16-18].

Bei COPD-Patienten gilt die Mangelernährung als bekannter eigenständiger Risikofaktor [19-22]. Während übergewichtige COPD-Patienten trotz gehäufter kardiovaskulärer Begleiterkrankungen eine längere Überlebenszeit zeigen, versterben normbis untergewichtige Patienten früher [23-25]. Insbesondere der Verlust an fettfreier Masse soll die Mortalitätsrate bei der COPD erhöhen $[23,26]$.

Vergleichbares gilt auch für den Zusammenhang zwischen Mangelernährung und der Überlebensrate nach Pneumonektomie $[27,28]$ beim Bronchialkarzinom.

Bislang wird in Deutschland eine relevante Mangelernährung bei stationären Patienten zumeist mit Hilfe des BMI dokumentiert. Erst bei einer manifesten Mangelernährung werden diese standardisiert zusatzernährt. Bei COPD-Patienten ließ sich im Anschluss an eine Ernährungstherapie kein positiver Effekt auf die Mortalität zeigen [29]. Trotzdem gibt es zunehmend Hinweise dafür, dass eine Intervention bei Mangelernährten in Bezug auf die Dauer des stationären Aufenthaltes und ihre Mortalität relevant ist [10].

Ziel dieser Arbeit war es, sowohl die Häufigkeit als auch das Risiko einer Mangelernährung zu überprüfen und miteinander zu vergleichen.

\section{Material und Methoden}

705 konsekutive Patienten (447 männlich, Alter 61,7 £13,4 Jahre, s. Tab. 1) wurden in unserer pneumologischen Fachklinik über drei Monate im Jahr 2009 hinsichtlich anthropometrischer Daten, BMI, ihrem Mangelernährungsrisiko (gemäß NRS), der Verweildauer und ihrer pneumologischen Grunderkrankung untersucht (s. $\odot$ Abb. 1).

Alle Patientendaten wurden mit Hilfe von geschultem ärztlichem und nicht-ärztlichem Personal erhoben. Die Patienten wurden gemäß ihrer pneumologischen Hauptdiagnose, die zur stationären Aufnahme führte, einer von vier Gruppen zugeordnet: schlafbezogenen Atmungsstörungen, Tumorerkrankungen, obstruktiven Atemwegserkrankungen sowie einer Gruppe mit allen weiteren Diagnosen. In letzterer wurden Patienten mit Pneumonien, interstitiellen Lungenerkrankungen, Tuberkulose und alle weiteren zusammengefasst.

Zur Beurteilung des Ernährungszustandes wurden der BMI und das NRS herangezogen.

Der BMI ist ein standardmäßig erfasster Parameter, für dessen Berechnung Körpergröße und Körpergewicht in Verhältnis gesetzt werden. Laut WHO wird ab einem BMI unter $18,5 \mathrm{~kg} / \mathrm{m}^{2}$ von einer Unterernährung gesprochen.

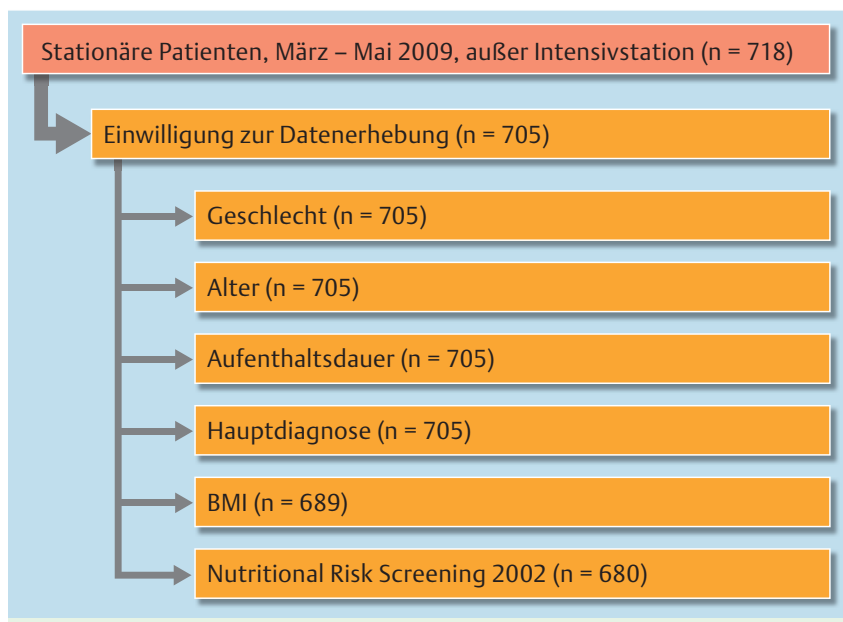

Abb. 1 Patienteneinschluss und erhobene Parameter. 


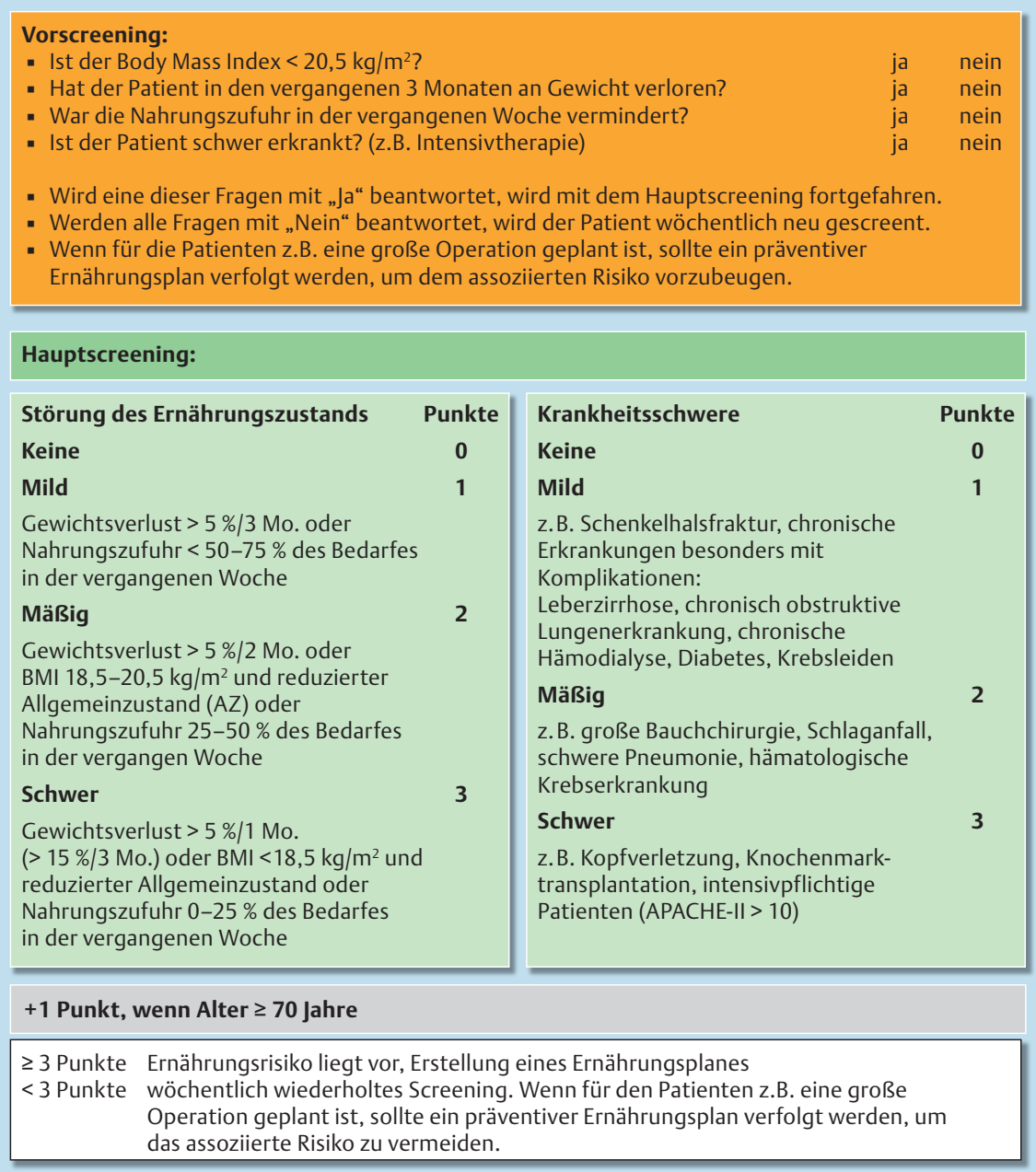

Abb.2 Nutritional Risk Screening (NRS) 2002, nach Kondrup J et al., Clinical Nutrition 2003; 22: 415-421. Empfohlen von der Europäischen Gesellschaft für Klinische Ernährung und Stoffwechsel (ESPEN). Für weitere Erklärungen und Details zur Anwendung des Fragebogens siehe bitte auch Originalartikel von Kondrup.

Das NRS 2002 (s. Abb.2) ist ein Screening-Instrument, das unter Berücksichtigung eines etwaigen Gewichtsverlustes innerhalb der letzten drei Monate, der Schwere weiterer Begleiterkrankungen sowie des Alters die Detektion eines ME-Risikos erlaubt. Dabei kann der Summenscore einen Wert von 0 bis 7 annehmen. Ab drei Punkten liegt ein Mangelernährungsrisiko vor. Im Vorscreening wird zunächst erfragt, ob ein BMI $<20,5 \mathrm{~kg} / \mathrm{m}^{2}$ vorliegt oder ein bekannter Grund zu einer Gewichtsreduktion führt. Falls eine dieser Fragen positiv beantwortet wird, folgt ein Hauptscreening. Dies teilt sich in drei Teile auf: die differenzierte Betrachtung des Gewichtsverlustes bzw. eine reduzierte Nahrungszufuhr (0 - 3 Punkte), die Schwere der Erkrankungen (0-3 Punkte) sowie das Lebensalter (0-1). Der Gewichtsverlust wird genau wie die Nahrungszufuhr erfragt oder aus den medizinischen Unterlagen erhoben.

Die Studie wurde von der Ethikkommission der Universität Witten/Herdecke positiv bewertet, und von den Patienten wurde ihr Einverständnis eingeholt.

Zur deskriptiven Auswertung der Ergebnisse wurden Anteile, Absolutwerte bzw. Mittelwerte mit Standardabweichung angegeben. Einflüsse wie die Faktoren Alter und Erkrankungsgruppe auf das Vorliegen eines Mangelernährungsrisikos gemäß NRS wurden mittels multipler logistischer Regression in SPSS 18 (SPSS Inc. Released 2009. PASW Statistics for Windows, Version 18.0. Chicago: SPPS Inc.) berechnet. Zum Vergleich zweier Mittelwerte wurde der t-Test verwendet.

\section{Ergebnisse}

Gemäß NRS bestand bei $14,3 \%$ der untersuchten Patienten ein Mangelernährungsrisiko (ME-Risiko), gemäß BMI (WHO) lag eine Unterernährung bei 2,5\% der Patienten vor (s. $\bullet$ Tab. 1).

Bei den über 65-jährigen lagen diese Werte bei 19,6\% (NRS) bzw. $1,5 \%$ (BMI). Es bestand kein relevanter Unterschied zwischen den Geschlechtern. Das Alter trug signifikant zum ME-Risiko bei (OR 1,054 pro zusätzlichem Lebensjahr, $\mathrm{p}<0,001$ ).

Während die meisten Patienten mit einer Pneumonie einen unauffälligen BMI aufwiesen (29 von 30 Patienten $\geq 18,5 \mathrm{~kg} / \mathrm{m}^{2}$ ), war das NRS bei sieben von 30 Patienten positiv. Ähnliches zeigte sich auch für Patienten mit obstruktiven Erkrankungen. Gemessen am BMI waren 6 von 174 Patienten (3\%) untergewichtig, gemäß NRS 28 von 174 (16\%) von einem ME-Risiko betroffen.

Eine vorliegende Tumorerkrankung erhöhte das ME-Risiko besonders stark (OR 2,33, p<0,001), während es bei schlafbezogenen Atmungsstörungen stark vermindert war (OR 0,04 p<0,001). Bei vorliegendem ME-Risiko war die Verweildauer mit 10,2 $\pm 9,5$ Tagen ( $n=97)$ gegenüber 5,4 $\pm 6,0$ Tagen bei Patienten ohne MERisiko ( $n=583$ ) signifikant erhöht $(p<0,001)$. Bei Ausschluss der Patienten mit einer schlafbezogenen Atmungsstörung blieb die Verweildauer weiterhin bei positivem NRS relevant erhöht $(12,0 \pm 15,5$ Tage mit 94 Patienten vs. 8,7 $\pm 7,0$ mit 311 Patienten, $\mathrm{p}=0,001)$. 


\section{Diskussion}

\section{$\nabla$}

Die vorliegende Studie bestätigt, dass auch in der Pneumologie ein relevanter Anteil der Patienten von einer Unterernährung oder dem Risiko einer Mangelernährung betroffen ist. Die enge Assoziation zwischen erhöhter Morbidität bzw. Mortalität und einer Mangelernährung wurde anhand des BMI für viele pneumologische Erkrankungen dokumentiert [1 -3].

In der vorliegenden Untersuchung wurde als Erweiterung zum BMI das NRS als Messinstrument zur Abschätzung einer drohenden Mangelernährung verwendet.

Diskussionsgegenstand ist, ob die Hinzunahme des NRS einen erheblichen Informationszugewinn auch für den Pneumologen beinhalten kann.

Bei der vorliegenden Klientel lag der Anteil an Unterernährten gemessen am BMI bei 2,5\%, gemessen am NRS bestand bei $14,3 \%$ der Patienten das Risiko einer ME. Das ist im Vergleich zur deutschen Multizenterstudie an hospitalisierten Patienten ein ähnlich hoher Wert in Bezug auf den BMI [3]. Pirlich et al. untersuchten in deutschen Kliniken die Mangelernährung, dies jedoch zu einem Zeitpunkt, zu dem das NRS noch nicht validiert war.

Die vorliegende Studie zeigt, dass die Anwendung des NRS im Gegensatz zur alleinigen Heranziehung des BMI auch diejenigen Patienten detektierten kann, die ein ME-Risiko aufweisen. Selbst wenn diese Grenze in Analogie zum Vorscreening-Abschnitt des NRS auf einen BMI von $20,5 \mathrm{~kg} / \mathrm{m}^{2}$ gesetzt würde, wären in der hier untersuchten Stichprobe mit 50/689 Patienten (7,3\%) lediglich halb so viele Patienten mittels BMI gefunden worden.

Eine kombinierte Betrachtung von BMI und NRS erscheint auch deswegen indiziert, da die europäische Gesellschaft für Ernährung (ESPEN) das NRS explizit zur Detektion eines Mangelernährungsrisikos empfiehlt (www.espen.org).

Pirlich et al. [3] zeigten einen erhöhten Anteil an Mangelernährung bei Frauen und bei älteren Patienten. In der hier untersuchten pneumologischen Patientengruppe entsprach das Alter einem signifikanten Risikofaktor einer Mangelernährung, die Geschlechtszugehörigkeit war unerheblich. Pirlich et al. untersuchten Patienten aus mehreren unterschiedlichen Kliniken. Es wurden gastroenterologische, chirurgische, geriatrische und gynäkologische Patienten hinsichtlich BMI untersucht. Männer und Frauen waren bei Pirlich et al. fast gleich verteilt $(\mathrm{m}=902, \mathrm{f}=984)$. Eine Erklärung für den höheren Anteil mangelernährter Patientinnen im Vergleich zu den Männern könnten die für diese Fachdisziplinen typischen Grunderkrankungen bieten. Als Beispiele sind Autoimmunerkrankungen oder Essstörungen zu nennen, die einerseits häufiger bei Frauen auftreten und zudem häufiger in der Gastroenterologie oder Allgemeinchirurgie als in der Pneumologie betreut werden. Da eine Differenzierung der jeweiligen Grunderkrankungen Pirlichs Artikel nicht zu entnehmen ist, bleibt die Ursache der überhäufig mangelernährten Frauen in Pirlichs Klientel hypothetisch.

Pirlichs Patienten waren im Durchschnitt 62,2 \pm 17,4 Jahre alt, unsere pneumologischen Patienten 61,7 $\pm 13,4$ Jahre. Die Vergleichbarkeit der untersuchten Patientengruppen erscheint daher hinsichtlich des Alters gegeben. Wie auch Pirlich et al. konnten wir das Alter als einen signifikanten Risikofaktor für eine Mangelernährung bestätigen.

Sowohl bei den Patienten mit einer Pneumonie als auch bei den Patienten mit einer chronisch obstruktiven Lungenerkrankung (COPD) lag in $23 \%$ bzw. 16\% ein ME-Risiko gemäß NRS vor. Untergewicht stellt einen relevanten Risikofaktor für die Entstehung einer ambulant erworbenen Pneumonie dar [16]. Es könnte da- her für die Sekundärprävention von Interesse sein, bereits das ME-Risiko eines Pneumoniepatienten festzustellen. Mittels BMI wird bei den Patienten formal ab einem Wert unter $18,5 \mathrm{~kg} / \mathrm{m}^{2}$ die Unterernährung eindeutig diagnostiziert. Bis zum Absinken auf einen solch niedrigen BMI-Wert mit einer Ernährungstherapie in der Nachsorge zu warten, ist im Zusammenhang mit dem Vorliegen einer Pneumonie zumindest diskussionswürdig.

Trotz allem kann der Eindruck entstehen, dass bei den pneumologischen Patienten ein ME-Risiko, das durch das NRS diagnostiziert wird, in seiner Relevanz überbewertet wird. Hiergegen sprechen die Validierung des NRS mit hoher Sensitivität und Spezifität sowie auch die langjährige Anwendung und Empfehlung durch die gastroenterologischen Fachgesellschaften [11,12]. Bei unseren pneumologischen Patienten wurde erwartet, dass die Tumorkranken überhäufig einen geringen BMI-Wert und ein Mangelernährungsrisiko aufwiesen. Des Weiteren wurde vermutet, dass bei den Patienten mit SBAS das Gegenteil der Fall sein müsste. Beides bestätigte sich in den Ergebnissen von NRS und BMI-Werten.

Es konnte bei unseren Patienten auch ein positiver Zusammenhang zwischen einem vorliegenden ME-Risiko gemäß NRS und der Dauer des Krankenhausaufenthaltes gezeigt werden. Ähnlich wie Lim et al. zeigte sich auch hier eine deutliche Verlängerung des Krankenhausaufenthaltes, wobei Lim et al. die Mangelernährung und nicht das Risiko einer Mangelernährung untersuchten [9].

Weiterhin ist der erzielte Informationsgewinn durch das NRS mit einer geringen Ressourcenbindung assoziiert. Bei uns konnte das NRS in 680 von 705 Fällen durch ärztliches sowie nicht-ärztliches Personal erhoben werden. Die hierfür nötige Datengewinnung ist nicht notgedrungen in den ärztlichen Aufgabenbereich zu legen. Die Fragen sind klar definiert und sicher durch geschultes Personal zu erheben. Eine Einbeziehung in den klinischen Alltag sollte daher problemlos erfolgen können.

Als Limitation der vorliegenden Studie muss erwähnt werden, dass keine longitudinalen Daten erhoben wurden. Daher kann nichts über den Verlauf oder den Vergleich zwischen ausschließlich beobachteten oder auch zusatzernährten NRS-positiven Patienten gesagt werden. Es wäre von großem Interesse zu wissen, ob eine enterale Zusatzernährung bei NRS-positiven Patienten einen positiven Effekt auf deren Morbidität oder Mortalität hätte. Es zeigt sich, dass mit Hilfe des NRS das Risiko einer ME differenzierter beurteilt werden kann und somit einen deutlichen Informationszugewinn für den Pneumologen bietet, der allein durch Betrachtung des BMI so nicht zu erreichen ist.

Das NRS kann also als ein probates und effizientes Mittel zur sensitiven Detektion eines Risikos für eine Mangelernährung in der Pneumologie angesehen werden.

\section{Danksagungen}

Unser Dank geht an unsere Kolleginnen Frau Dr. Natalie Funke und Frau Lovorka Kostovic, die die Daten maßgeblich für die vorliegende Studie erhoben haben.

\section{Interessenkonflikt \\ $\nabla$}

Die Autoren geben an, dass kein Interessenkonflikt besteht. 


\section{Literatur}

1 Coats KG, Morgan SL, Bartolucci AA et al. Hospital-associated malnutrition: a reevaluation 12 years later. J Am Diet Assoc 1993; 93: 27-33

2 Kondrup J, Rasmussen $\mathrm{HH}$, Hamberg $\mathrm{O}$ et al. Nutritional risk screening (NRS 2002): a new method based on an analysis of controlled clinical trials. Clin Nutr 2003; 22: $321-336$

3 Pirlich M, Schutz T, Norman K et al. The German hospital malnutrition study. Clin Nutr 2006; 25: 563-572

4 Waitzberg DL, Caiaffa WT, Correia MI. Hospital malnutrition: the Brazilian national survey (IBRANUTRI): a study of 4000 patients. Nutrition 2001; $17: 573-580$

5 Kruizenga HM, Wierdsma NJ, van Bokhorst MA et al. Screening of nutritional status in The Netherlands. Clin Nutr 2003; 22: 147-152

6 Norman K, Pichard C, Lochs H et al. Prognostic impact of disease-related malnutrition. Clin Nutr 2008; 27: 5-15

7 Kyle UG, Pirlich M, Schuetz T et al. Is nutritional depletion by Nutritional Risk Index associated with increased length of hospital stay? A population-based study. JPEN J Parenter Enteral Nutr 2004; 28: 99-104

8 Ockenga J, Freudenreich M, Zakonsky $R$ et al. Nutritional assessment and management in hospitalised patients: implication for DRG-based reimbursement and health care quality. Clin Nutr 2005; 24: 913-919

$9 \mathrm{Lim}$ SL, Ong KC, Chan YH et al. Malnutrition and its impact on cost of hospitalization, length of stay, readmission and 3-year mortality. Clin Nutr 2012; 31: $345-350$

10 Kitson AL, Schultz TJ, Long $L$ et al. The prevention and reduction of weight loss in an acute tertiary care setting: protocol for a pragmatic stepped wedge randomised cluster trial (the PRoWL project). BMC Health Serv Res 2013; 13: 299

11 Almeida AI, Correia M, Camilo M et al. Nutritional risk screening in surgery: valid, feasible, easy! Clin Nutr 2012; 31: 206-211

12 Rasmussen $\mathrm{HH}$, Holst M, Kondrup J. Measuring nutritional risk in hospitals. Clin Epidemiol 2010; 2: 209-216

13 Kollef MH, Shorr A, Tabak YP et al. Epidemiology and outcomes of health-care-associated pneumonia: results from a large US database of culture-positive pneumonia. Chest 2005; 128: 3854-3862

14 Melsen WG, Rovers MM, Koeman M et al. Estimating the attributable mortality of ventilator-associated pneumonia from randomized prevention studies. Crit Care Med 2011; 39: 2736-2742

15 American Thoracic S, Infectious Diseases Society of A. Guidelines for the management of adults with hospital-acquired, ventilator-associated, and healthcare-associated pneumonia. Am J Respir Crit Care Med $2005 ; 171: 388-416$
16 Almirall J, Bolibar I, Serra-Prat $M$ et al. New evidence of risk factors for community-acquired pneumonia: a population-based study. Eur Respir J 2008; 31: $1274-1284$

17 Almirall J, Bolibar I, Balanzo X et al. Risk factors for community-acquired pneumonia in adults: a population-based case-control study. Eur Respir J 1999; 13: 349-355

18 Corrales-Medina VF, Valayam J, Serpa JA et al. The obesity paradox in community-acquired bacterial pneumonia. Int J Infect Dis 2011; 15: e54-57

19 Celli BR, Cote CG, Lareau SC et al. Predictors of Survival in COPD: more than just the FEV1. Respir Med 2008; 102 (Suppl. 01): S27-S35

20 Yang $L$, Zhou $M$, Smith $M$ et al. Body mass index and chronic obstructive pulmonary disease-related mortality: a nationally representative prospective study of 220,000 men in China. Int J Epidemiol 2010; 39: $1027-1036$

21 Breeze E, Clarke R, Shipley MJ et al. Cause-specific mortality in old age in relation to body mass index in middle age and in old age: follow-up of the Whitehall cohort of male civil servants. Int J Epidemiol 2006; 35: $169-178$

22 Mannino DM, Buist AS, Petty TL et al. Lung function and mortality in the United States: data from the First National Health and Nutrition Examination Survey follow up study. Thorax 2003; 58: 388-393

23 Schols AM, Broekhuizen R, Weling-Scheepers CA et al. Body composition and mortality in chronic obstructive pulmonary disease. Am J Clin Nutr 2005; 82: 53-59

24 Landbo C, Prescott E, Lange P et al. Prognostic value of nutritional status in chronic obstructive pulmonary disease. Am J Respir Crit Care Med 1999; 160: $1856-1861$

25 Schols AM, Slangen J, Volovics L et al. Weight loss is a reversible factor in the prognosis of chronic obstructive pulmonary disease. Am J Respir Crit Care Med 1998; 157: 1791-1797

26 Schols AM. Nutritional rehabilitation: from pulmonary cachexia to sarcoPD. Eur Respir J 2009; 33: 949-950

27 Bagan P, Berna P, De Dominicis F et al. Nutritional status and postoperative outcome after pneumonectomy for lung cancer. Ann Thorac Surg 2013; 95: $392-396$

28 Sanchez-Lara K, Turcott JG, Juarez E et al. Association of nutrition parameters including bioelectrical impedance and systemic inflammatory response with quality of life and prognosis in patients with advanced non-small-cell lung cancer: a prospective study. Nutr Cancer 2012; 64: 526-534

29 Itoh $M$, Tsuji T, Nemoto $K$ et al. Undernutrition in patients with COPD and its treatment. Nutrients 2013; 5: 1316-1335 